\title{
Urinary neutrophil gelatinase-associated lipocalin values alone and combined with Prognostic Index predict septic AKI, DIC, and shock: a pilot study
}

\author{
Yuichiro Shimoyama ${ }^{1}$, Osamu Umegaki ${ }^{1}$, Noriko Kadono ${ }^{1}$ and Toshiaki Minami ${ }^{2}$
}

\begin{abstract}
Objective: Sepsis is a syndrome involving life-threatening organ dysfunction. The present study aimed to determine whether septic AKI, ARDS, DIC, and shock can be predicted more readily by combining UNGAL values and inflammation-based prognostic scores, over the use of UNGAL values alone.

Results: ROC curve analyses yielded the following cut-off values: AKI: 438.5 (ng/ml) for UNGAL at Day 1 (AUC, 0.8), 476.9 (ng/ml) for UNGAL at Day 2 (AUC, 0.86), $123.8(\mathrm{ng} / \mathrm{ml}$ ) for UNGAL at Day 3 (AUC, 0.81), $133.6(\mathrm{ng} / \mathrm{ml})$ for UNGAL at Day 4 (AUC, 0.78), 1.0 for iNS NGAL-NLR (AUC, 0.75), 2.0 for iNS NGAL-PI (AUC, 0.77), DIC; 648.5 (ng/ml) for uNGAL at Day 1 (AUC, 0.77); shock; 123.8 (ng/ml) for uNGAL at Day 3 (AUC, 0.71) and 9 for SOFA (AUC, 0.71). Multivariate logistic regression analyses revealed iNS NGAL-PI to be a significant independent predictor of AKI $(\mathrm{OR}, 20.62 ; 95 \% \mathrm{Cl}, 1.03-$ 412.3; $p=0.048$ ).
\end{abstract}

Keywords: Neutrophil gelatinase-associated lipocalin, Sepsis, Sepsis-3 definition, Inflammation-based prognostic scores, Organ dysfunction

\section{Introduction}

In 2016, the Third International Consensus Definitions Task Force (Sepsis-3) proposed new criteria for defining sepsis [1]. Sepsis is a syndrome involving life-threatening organ dysfunction caused by a dysregulated host response to infection. In sepsis patients, an increase in the Sequential Organ Failure Assessment (SOFA) score by $\geq 2$ is associated with in-hospital mortality as high as $10 \%$ [1].

Neutrophil gelatinase-associated lipocalin (NGAL) is involved in metabolic homeostasis, apoptosis, infection, immune response, and inflammation, and is a

\footnotetext{
*Correspondence: shimocchiliebesfreud512@yahoo.co.jp

${ }^{1}$ Department of Anesthesiology, Osaka Medical College, Intensive Care Unit, Osaka Medical College Hospital, 2-7 Daigaku-machi, Takatsuki, Osaka 569-8686, Japan

Full list of author information is available at the end of the article
}

potential clinical biomarker for the onset and progression of inflammatory diseases, including arthritic diseases, severe acute pancreatitis, obesity-related metabolic diseases, cardiovascular diseases, AKI, lupus nephritis, intestinal inflammation, and multiple sclerosis $[2,3]$. Inflammation-based prognostic scores including the Glasgow Prognostic Score (GPS; calculated based on serum C-reactive protein (CRP) and albumin levels), neutrophil to lymphocyte ratio (NLR), platelet to lymphocyte ratio (PLR), Prognostic Nutritional Index (PNI; calculated based on albumin and lymphocyte counts), and Prognostic Index (PI; calculated based on serum CRP and white blood cell counts) are prognostic biomarkers for several types of cancer [4].

No study to date has assessed the association of septic AKI, ARDS, DIC, and shock with uNGAL values alone or in combination with inflammation-based prognostic 
scores (i.e., GPS, NLR, PLR, PNI, and PI) in patients with sepsis as defined by the Sepsis-3 definition. Accordingly, the present study aimed to test the following hypotheses: (1) UNGAL values are useful predictors of septic AKI, ARDS, DIC, and shock in sepsis patients, (2) uNGAL values are superior to inflammation-based prognostic scores for predicting septic AKI, ARDS, DIC, and shock, and (3) the ability to predict septic AKI, ARDS, DIC, and shock can be improved by combining uNGAL values and inflammation-based prognostic scores relative to uNGAL values alone.

\section{Main text \\ Methods}

\section{Patients and study design}

This single-center prospective study was conducted in a 16-bed ICU. The study protocol was approved by the Ethics Committee of Osaka Medical College (Osaka, Japan). Adult patients diagnosed with sepsis according to the Sepsis-3 definition [1] and admitted to the ICU were prospectively examined from June 2018 to November 2018. Informed consent was obtained from all patients enrolled in this study or their families. Exclusion criteria were as follows: (1) patients with unstable renal function (increase of $\mathrm{sCr}$ by $\geq 0.2 \mathrm{mg} / \mathrm{dl}$ in the past month); (2) patients who died within $24 \mathrm{~h}$ after being admitted to the ICU; (3) patients on dialysis; (4) patients aged $\leq 18$ years, (5) patients who had an immunosuppressive disease (e.g., HIV); and (6) patients undergoing immunosuppressive therapy (e.g., chemotherapy, chronic steroid use, autoimmune disease treatment) within one month of the study.

Urine samples were collected from each patient with a urethral balloon bag to measure urinary NGAL (uNGAL) after ICU entry at the following time points: immediately after and 2, 3, and 4 days after ICU entry. Septic AKI was defined as stage $\geq 1$ kidney disease according to the Kidney Disease: Improving Global Outcomes (KDIGO) classification [5]. Septic ARDS was defined according to the Berlin definition [6]. Septic DIC was defined according to the Japanese Association for Acute Medicine DIC diagnostic criteria [7]. Septic shock was defined according to the Sepsis-3 definition [1]. GPS, NLR, PLR, PI, and PNI were examined immediately after ICU entry. For category classification, a total score was calculated (hereafter, "inflammation-NGAL score [iNS]") as follows: a score of 1 was assigned if UNGAL values and inflammation-based prognostic scores immediately after ICU entry were above cut-offs determined by ROC curve analysis for 28-day mortality; a score of 0 was assigned if the values were below the cut-offs (total score range, $0-2$ points). As an example of nomenclature, the combination of UNGAL and NLR is presented as "iNS NGAL-NLR." uNGAL, inflammation-based prognostic scores, iNS, and changes $(\Delta)$ in uNGAL relative to uNGAL values immediately after ICU entry at each sampling point were compared between patients with septic AKI, ARDS, DIC, or shock after ICU entry and those without these disorders.

\section{Laboratory assessments}

Urine samples were centrifuged at $3500 \mathrm{rpm}$ for $8 \mathrm{~min}$ and stored at $4{ }^{\circ} \mathrm{C}$ within $12 \mathrm{~h}$ after patient enrollment. uNGAL was measured using the ARCHITECT platform (Abbott Japan Co., Ltd., Tokyo, Japan) [8], a chemiluminescent microparticle immunoassay using a noncompetitive, 2-antianalyte antibody sandwich. In this assay, a microparticle

\section{Table 1 Baseline demographic characteristics}

\begin{tabular}{|c|c|}
\hline Variable & $n=44$ \\
\hline Age (years) & $74.0(63.8-78.8)$ \\
\hline Gender (male) (\%) & $29(65.9)$ \\
\hline Cancer (\%) & $27(61.4)$ \\
\hline Coronary artery disease (\%) & $1(2.3)$ \\
\hline Diabetes mellitus (\%) & $4(9.1)$ \\
\hline Hypertension (\%) & $10(25.0)$ \\
\hline Albumin (g/dL) & $2.3(1.8-3.1)$ \\
\hline $\mathrm{CRP}(\mathrm{mg} / \mathrm{dL})$ & $9.3(2.5-19.0)$ \\
\hline WBC $\left(\times\left. 10^{9}\right|^{-1}\right)$ & $11.8(5.7-17.8)$ \\
\hline Neutrophil count $\left(\times\left. 10^{9}\right|^{-1}\right)$ & $8.8(2.9-15.7)$ \\
\hline Lymphocyte count $\left(\times\left. 10^{9}\right|^{-1}\right)$ & $0.5(0.2-0.7)$ \\
\hline Platelet count $\left(\times 10^{4} \mathrm{~mm}^{-3}\right)$ & $17.8(11.4-25.3)$ \\
\hline Fibrinogen (mg/dL) & $537(318-730)$ \\
\hline Survival (deceased) (\%) & $12(27.3)$ \\
\hline Septic AKI (\%) & $20(45.5)$ \\
\hline Septic ARDS (\%) & $5(11.4)$ \\
\hline Septic shock (\%) & $27(61.4)$ \\
\hline Septic DIC (\%) & $15(34.1)$ \\
\hline NGAL: Day 1 (ng/mL) & $247.9(77.1-634.6)$ \\
\hline NGAL: Day 2 (ng/mL) & $268.9(127.9-1098.9)$ \\
\hline NGAL: Day 3 (ng/mL) & $123.8(63.1-461.7)$ \\
\hline NGAL: Day 4 (ng/mL) & $120.7(50.2-638.1)$ \\
\hline$\triangle \mathrm{NGAL}$ : Day 2-Day 1 (ng/mL) & $0.5(-140.6-129.4)$ \\
\hline$\triangle \mathrm{NGAL}$ : Day 3-Day 1 (ng/mL) & $-10.6(-198.2-90.7)$ \\
\hline$\triangle \mathrm{NGAL}:$ Day 4-Day 1 (ng/mL) & $-14.75(-312.4-86.3)$ \\
\hline GPS & $1(1-2)$ \\
\hline NLR & $17.7(5.3-37.7)$ \\
\hline PLR & $373.0(195.7-540.9)$ \\
\hline PI & $1(0-2)$ \\
\hline $\mathrm{PNI}$ & $26.5(20.2-33.6)$ \\
\hline SOFA & $9(6-11)$ \\
\hline qSOFA & $2(1-3)$ \\
\hline
\end{tabular}

CRP C-reactive protein, WBC white blood cell, AKI acute kidney injury, ARDS acute respiratory distress syndrome, DIC disseminated intravascular coagulation, NGAL neutrophil gelatinase-associated lipocalin, GPS Glasgow prognostic score, NLR neutrophil to lymphocyte ratio, $P L R$ platelet to lymphocyte ratio, $P I$ prognostic index, $P N /$ prognostic nutritional index, qSOFA quick Sequential Organ Failure Assessment 
reagent is prepared by covalently attaching an anti-analyte antibody to paramagnetic particles, and a conjugate reagent is prepared by labeling a second anti-analyte antibody with acridinium. The calibrator used for the UNGAL assay was recombinant uNGAL; the highest calibrator sample was $1500 \mathrm{ng} / \mathrm{ml} \mathrm{uNGAL}$, with a coefficient of variation of $3.0 \%$ at $385 \mathrm{ng} / \mathrm{ml}$ [8]. Co-investigators who performed the biomarker assays were blinded to AKI onset.

\section{Statistical analysis}

Categorical data are reported as percentages and compared using Fisher's exact test. Continuous data are reported as medians with inter-quartile ranges and compared using the Mann-Whitney U test. Receiver operating characteristics (ROC) curves were generated for uNGAL, inflammation-based prognostic scores, iNS, and $\triangle$ uNGAL, and areas under the curve (AUCs), cut-off values, sensitivities, and specificities were calculated. With the exceptions of Sequential Organ Failure Assessment (SOFA) and quick SOFA (qSOFA), variables which could be obtained immediately after ICU entry (with $\mathrm{P}<0.05$ in univariate analysis) were examined further by multivariate logistic regression analysis for septic AKI, ARDS, DIC, and shock. Results of these analyses were then compared with results of multivariate logistic regression analysis performed with SOFA and qSOFA as explanatory variables. $\mathrm{P}<0.05$ was considered statistically significant. JMP software version 11.0.0 (SAS Institute Inc., NC, USA) was used for all statistical analyses.

\section{Results}

Baseline characteristics of patients are shown in Table 1. No significant differences were observed in age and gender for septic AKI, ARDS, DIC, and shock groups (data not shown).
ROC curve analyses (Table 2) yielded the following cutoff values: AKI: $438.5(\mathrm{ng} / \mathrm{ml})$ for uNGAL at Day 1 (AUC, 0.8; sensitivity, 65\%; specificity, 83\%), $476.9(\mathrm{ng} / \mathrm{ml})$ for uNGAL at Day 2 (AUC, 0.86; sensitivity, 71\%; specificity, $91 \%), 123.8(\mathrm{ng} / \mathrm{ml}$ ) for uNGAL at Day 3 (AUC, 0.81 ; sensitivity, 92\%; specificity, 74\%), 133.6 (ng/ml) for uNGAL at Day 4 (AUC, 0.78; sensitivity, 67\%; specificity, 71\%), 1.0 for iNS NGAL-NLR (AUC, 0.75; sensitivity, 75\%; specificity, 75\%), 2.0 for iNS NGAL-PI (AUC, 0.77; sensitivity, 50\%; specificity, 92\%),DIC; 648.5 (ng/ml) for uNGAL at Day 1 (AUC, 0.77; sensitivity, 60\%; specificity, 97\%); shock; 123.8 (ng/ml) for uNGAL at Day 3 (AUC, 0.71; sensitivity, 68.4\%; specificity, 75\%) and 9 for SOFA (AUC, 0.71; sensitivity, $73.1 \%$; specificity, $64.7 \%$ ).

Data on variables which could be obtained immediately after ICU entry and were significant $(\mathrm{P}<0.05)$ in univariate analysis (Table 3 ) were examined further by multivariate logistic regression analysis for septic AKI, ARDS, DIC, and shock. The analyses revealed iNS NGAL-PI to be a significant independent predictor of AKI (OR, 20.62; 95\% CI, 1.03-412.3; $\mathrm{p}=0.048$; Additional file 1: Table S1). On the other hand, uNGAL alone was not identified as a significant independent predictor by multivariate logistic regression analysis.

\section{Discussion}

ROC curve analyses identified the following variables to be predictors of septic AKI, DIC, and shock: UNGAL at Days 1-4, iNS NGAL-NLR, and iNS-PI for septic AKI; uNGAL Day 1 for septic DIC; and uNGAL Day 3 and SOFA for septic shock (Table 2). Multivariate analysis revealed iNS NGAL-PI to be a predictor for septic AKI (Additional file 1: Table S1). On the other hand, inflammation-based prognostic scores, when used alone, did

Table 2 Receiver operating curve analysis

\begin{tabular}{|c|c|c|c|c|c|}
\hline Variable & AUC & Cut-off & $P$ value & Sensitivity (\%) & Specificity (\%) \\
\hline \multicolumn{6}{|l|}{ Septic AKI } \\
\hline $\operatorname{NGAL}(\mathrm{ng} / \mathrm{mL})$ Day 1 & 0.80 & 438.50 & $<0.001$ & 65.0 & 83.3 \\
\hline $\operatorname{NGAL}(\mathrm{ng} / \mathrm{mL})$ Day 2 & 0.86 & 476.90 & $<0.001$ & 70.6 & 90.5 \\
\hline NGAL $(n g / m L)$ Day 3 & 0.81 & 123.80 & $<0.001$ & 91.7 & 73.7 \\
\hline NGAL $(n g / m L)$ Day 4 & 0.78 & 133.60 & 0.002 & 66.7 & 70.6 \\
\hline iNS NGAL-NLR & 0.75 & 1.00 & $<0.001$ & 75.0 & 75.0 \\
\hline iNS NGAL-PI & 0.77 & 2.00 & $<0.001$ & 50.0 & 91.7 \\
\hline \multicolumn{6}{|l|}{ Septic DIC } \\
\hline $\operatorname{NGAL}(\mathrm{ng} / \mathrm{mL})$ Day 1 & 0.77 & 648.50 & 0.001 & 60.0 & 96.6 \\
\hline \multicolumn{6}{|l|}{ Septic shock } \\
\hline NGAL (ng/mL) Day 3 & 0.71 & 123.80 & 0.031 & 68.4 & 75.0 \\
\hline SOFA & 0.71 & 9.00 & 0.009 & 73.1 & 64.7 \\
\hline
\end{tabular}

$A U C$ area under the curve, AKI acute kidney injury, NGAL neutrophil gelatinase-associated lipocalin, iNS inflammation-NGAL score, NLR neutrophil to lymphocyte ratio, $P I$ prognostic index, DIC disseminated intravascular coagulation, SOFA Sequential Organ Failure Assessment 
Table 3 Predictive factors for septic AKI, ARDS, Shock, and DIC (univariate analysis)

\begin{tabular}{|c|c|c|c|c|}
\hline Variable & $\begin{array}{l}\text { AKI }(n=20) \\
P \text { value }\end{array}$ & $\begin{array}{l}\operatorname{ARDS}(\mathrm{n}=5) \\
P \text { value }\end{array}$ & $\begin{array}{l}\text { Shock }(n=27) \\
P \text { value }\end{array}$ & $\begin{array}{l}\text { DIC }(n=15) \\
P \text { value }\end{array}$ \\
\hline Age & 0.822 & 0.335 & 0.708 & 0.264 \\
\hline Gender & 0.246 & 0.480 & 1.000 & 1.000 \\
\hline Cancer & 0.651 & 0.359 & 0.526 & 0.333 \\
\hline Coronary artery disease & 0.356 & 1.000 & 0.386 & 1.000 \\
\hline Diabetes mellitus & 0.213 & 0.394 & 0.634 & 1.000 \\
\hline Hypertension & 0.036 & 0.586 & 0.158 & 1.000 \\
\hline Albumin & 0.723 & 0.810 & 0.141 & 0.275 \\
\hline CRP & 0.038 & 0.671 & 0.588 & 0.116 \\
\hline WBC & 0.120 & 0.365 & 0.152 & 0.328 \\
\hline Neutrophil & 0.120 & 0.385 & 0.233 & 0.665 \\
\hline Lymphocytes & 0.706 & 0.839 & 0.057 & 0.012 \\
\hline Platelet count & 0.540 & 0.739 & 0.177 & 0.000 \\
\hline Fibrinogen & 0.448 & 0.825 & 0.043 & 0.072 \\
\hline Survival & 0.103 & 0.116 & 0.090 & 0.284 \\
\hline Septic AKI & & 0.160 & 0.124 & 0.059 \\
\hline Septic ARDS & 0.099 & & 0.634 & 1.000 \\
\hline Septic shock & 0.090 & 0.634 & & 0.021 \\
\hline Septic DIC & 0.042 & 1.000 & 0.021 & \\
\hline NGAL: Day 1 & 0.001 & 0.189 & 0.049 & 0.003 \\
\hline NGAL: Day 2 & 0.000 & 0.401 & 0.120 & 0.054 \\
\hline NGAL: Day 3 & 0.004 & 0.547 & 0.057 & 0.139 \\
\hline NGAL: Day 4 & 0.019 & 0.773 & 0.096 & 0.157 \\
\hline$\triangle \mathrm{NGAL}:$ Day 2-Day 1 & 0.736 & 0.267 & 0.794 & 0.332 \\
\hline$\triangle \mathrm{NGAL}$ : Day 3-Day 1 & 0.351 & 0.423 & 0.417 & 0.459 \\
\hline$\triangle \mathrm{NGAL}:$ Day 4-Day 1 & 0.571 & 0.962 & 0.824 & 0.707 \\
\hline GPS & 0.390 & 0.709 & 0.935 & 0.222 \\
\hline NLR & 0.300 & 0.471 & 0.990 & 0.480 \\
\hline PLR & 0.572 & 0.753 & 0.691 & 0.304 \\
\hline PI & 0.084 & 0.596 & 0.299 & 0.885 \\
\hline $\mathrm{PNI}$ & 0.860 & 0.725 & 0.101 & 0.113 \\
\hline iNS-GPS & 0.024 & 0.158 & 0.688 & 0.045 \\
\hline iNS- NLR & 0.001 & 0.756 & 0.115 & 0.021 \\
\hline iNS- PLR & 0.022 & 0.472 & 0.284 & 0.492 \\
\hline iNS- PI & 0.001 & 0.264 & 0.308 & 0.064 \\
\hline iNS- PNI & 0.043 & 0.419 & 0.840 & 0.273 \\
\hline SOFA & 0.723 & 0.568 & 0.020 & 0.348 \\
\hline qSOFA & 0.294 & 0.493 & 0.309 & 0.508 \\
\hline
\end{tabular}

$A K I$ acute kidney injury, $A R D S$ acute respiratory distress syndrome, DIC disseminated intravascular coagulation, $C R P C$-reactive protein, WBC white blood cell, NGAL neutrophil gelatinase-associated lipocalin, GPS Glasgow Prognostic Score, NLR neutrophil to lymphocyte ratio, PLR platelet to lymphocyte ratio, PI prognostic index, $P N I$ prognostic nutritional index, iNS inflammation-NGAL score, qSOFA quick Sequential Organ Failure Assessment

not serve as significant prognostic predictors based on AUC, sensitivity, and specificity determined by ROC curve analysis and univariate analysis (data not shown).

Sepsis involves the activation of both pro- and antiinflammatory responses [9], with modifications in non-immunologic pathways including cardiovascular, neuronal, autonomic, hormonal, bioenergetic, metabolic, and coagulation pathways [10-12]]. NGAL is associated with a variety of physiological and pathophysiological processes including metabolic homeostasis, apoptosis, infection, immune response, and inflammation [3]. Vanmassenhove et al. reported that serum NGAL levels increase in parallel with the severity of sepsis, illness, and inflammation in sepsis patients [13]. The present study found that UNGAL can serve as a biomarker for various complications related to sepsis, including not only septic 
AKI, but also septic DIC and shock. This likely involves the above-mentioned characteristics of NGAL.

The AUC, sensitivity, and specificity of iNS NGAL-PI for predicting AKI were $0.769,50$, and $92 \%$, respectively, corresponding to a higher specificity than those observed for uNGAL Days 1-4 alone. These findings suggest that combining PI with UNGAL values may serve as an easy "rule in" test. The AUC, sensitivity, and specificity of iNS NGAL-PI were also higher than those of SOFA and qSOFA (data not shown). Multivariate analyses revealed that higher iNS NGAL-PI values corresponded to a stronger ability to predict AKI $(\mathrm{OR}, 20.62 ; \mathrm{p}=0.048)$ (Table S1), and that iNS NGAL-PI more accurately predicted AKI than UNGAL alone, SOFA, and qSOFA in sepsis patients. The advantage of combining biomarkers to improve their performance for predicting AKI has been reported in previous studies [14-16]. In those studies, urinary biomarkers that reflect renal tubular injury such as NGAL, kidney injury molecule-1, L-type fatty acid-binding protein, and $\mathrm{N}$-acetyl- $\beta$-D-glucosaminidase were combined to predict post-cardiac surgery AKI. As mention above, sepsis and septic AKI are highly complex and involve multiple pathophysiological mechanisms [17]. For such complex conditions, combinations of biomarkers involved in different pathways may help make accurate predictions at an early stage.

We previously reported that NLR is superior to other inflammation-based prognostic scores in predicting the mortality of patients with gastrointestinal perforation and pneumonia $[18,19]$. In the context of AKI, however, the present study found PI to be a predictor of septic AKI. PI reportedly predicts the prognosis of patients with lung cancer [20]]. According to a study by Proctor et al., which involved comparing the prognostic value of numerous inflammation-based prognostic scores (modified GPS, NLR, PLR, PI, and PNI) for various cancers, modified GPS and PI were found to have prognostic value for cancer independent of the tumor site [21]. Our findings suggest that, even among the various variables which can be tested to assess inflammation, CRP and lymphocytes (which are used to calculate PI) are important for diagnosing septic AKI.

No previous study has assessed the association between uNGAL alone or UNGAL combined with PI and septic AKI, DIC, and shock in patients with sepsis defined according to the Sepsis-3 definition. Our findings are novel in this respect. Further studies will be needed to clarify the mechanism underlying the predictive value of uNGAL and PI for septic AKI.

\section{Conclusion}

In conclusion, uNGAL and iNS NGAL-PI were found to be predictors of septic AKI, DIC, and shock. Combining PI with uNGAL values improved the predictive value of uNGAL for septic AKI. Moreover, iNS NGALPI was found to more strongly predict septic AKI in sepsis patients than SOFA and qSOFA. Further studies aimed at understanding the exact role of uNGAL and PI in predicting septic AKI, DIC, and shock are warranted.

\section{Limitation}

This study has several limitations. First, this was a single-center study with a small cohort. Second, this study used a single biomarker, and no comparisons were made with other biomarkers.

\section{Supplementary information}

Supplementary information accompanies this paper at https://doi. org/10.1186/s13104-020-05232-w.

Additional file 1: Table S1. Predictive Factors for Septic AKI, ARDS, Shock, and DIC (Multivariate Analysis).

\section{Abbreviations}

SOFA: The Sequential Organ Failure Assessment; NGAL: Neutrophil gelatinaseassociated lipocalin; GPS: Glasgow Prognostic Score; CRP: C-reactive protein (CRP); NLR: Neutrophil to lymphocyte ratio; PLR: Platelet to lymphocyte ratio; PNI: Prognostic Nutritional Index; PI: Prognostic Index; ROC: Receiver operating characteristics; AUC: Areas under the curve; iNS: Inflammation-NGAL score.

\section{Acknowledgements}

Not applicable.

\section{Authors' contributions}

YS conceived and designed the study, collected the data, performed the formal analysis and interpretation, drafted the manuscript, and managed revisions. OU, NK and TM contributed to the concept and design, as well as critical revision of the manuscript. All authors read and approved the final manuscript.

\section{Funding}

This research did not receive any specific grant from funding agencies in the public, commercial, or not-for-profit sectors.

\section{Availability of data and materials}

The datasets used and/or analyzed during the current study are available from the corresponding author on reasonable request.

Ethics approval and consent to participate

The study protocol was approved by the Ethics Committee of Osaka Medical College (Osaka, Japan). Written informed consent was obtained from all patients enrolled in this study or their families.

\section{Consent for publication}

Not applicable. 


\section{Competing interests}

The authors declare that they have no competing interests.

\section{Author details}

${ }^{1}$ Department of Anesthesiology, Osaka Medical College, Intensive Care Unit, Osaka Medical College Hospital, 2-7 Daigaku-machi, Takatsuki, Osaka 569-8686, Japan. ${ }^{2}$ Department of Anesthesiology, Osaka Medical College, Osaka Medical College Hospital, Takatsuki, Japan.

Received: 3 July 2020 Accepted: 13 August 2020

Published online: 18 August 2020

\section{References}

1. Singer M, Deutschman CS, Seymour CW, Shankar-Hari M, Annane D, Bauer $M$, et al. The third international consensus definitions for sepsis and septic shock (Sepsis-3). JAMA. 2016:315:801-10.

2. Devarajan P. NGAL in acute kidney injury: from serendipity to utility. Am J Kidney Dis. 2008;52:395-9.

3. Abella V, Scotece M, Conde J, Gómez R, Lois A, Pino J, et al. The potential of lipocalin-2/NGAL as biomarker for inflammatory and metabolic diseases. Biomarkers. 2015;20:565-71.

4. Kinoshita A, Onoda H, Imai N, Iwaku A, Oishi M, Fushiya N, et al. Comparison of the prognostic value of inflammation-based prognostic scores in patients with hepatocellular carcinoma. Br J Cancer. 2012;107:988-93.

5. KDIGO Guideline. Kidney disease: improving global outcomes. Clinical practice guideline on acute kidney injury. 2011. http://www.kdigo.org. Accessed 4 Apr 2012

6. ARDS Definition Task Force, Ranieri VM, Rubenfeld GD, Thompson BT, Ferguson ND, Caldwell E, Fan E, et al. Acute respiratory distress syndrome: the Berlin Definition. JAMA. 2012;307:2526-33.

7. Iba T, Umemura Y, Watanabe E, Wada T, Hayashida K, Kushimoto S, Japanese Surviving Sepsis Campaign Guideline Working Group for disseminated intravascular coagulation. Diagnosis of sepsis-induced disseminated intravascular coagulation and coagulopathy. Acute Med Surg. 2019:6:223-32.

8. Grenier FC, Ali S, Syed H, Workman R, Martens F, Liao M, et al. Evaluation of the ARCHITECT urine NGAL assay: assay performance, specimen handling requirements and biological variability. Clin Biochem. 2010;43:615-20

9. Hotchkiss RS, Monneret G, Payen D. Sepsis-induced immunosuppression: from cellular dysfunctions to immunotherapy. Nat Rev Immunol. 2013;13(12):862-74.
10. Angus DC, van der Poll T. Severe sepsis and septic shock. N Engl J Med. 2013;369(9):840-51.

11. Deutschman CS, Tracey KJ. Sepsis: current dogma and new perspectives. Immunity. 2014;40(4):463-75.

12. Singer $M$, De Santis V, Vitale $D$, Jeffcoate W. Multiorgan failure is an adaptive, endocrine mediated, metabolic response to overwhelming systemic inflammation. Lancet. 2004:364(9433):545-8.

13. Vanmassenhove J, Glorieux G, Lameire N, Hoste E, Dhondt A, Vanholder R, et al. Influence of severity of illness on neutrophil gelatinase-associated lipocalin performance as a marker of acute kidney injury: a prospective cohort study of patients with sepsis. BMC Nephrol. 2015;16:18.

14. Katagiri D, Doi K, Honda K, Negishi K, Fujita T, Hisagi M, et al. Combination of two urinary biomarkers predicts acute kidney injury after adult cardiac surgery. Ann Thorac Surg. 2012;93:577-83.

15. Han WK, Wagener G, Zhu Y, Wang S, Lee HT. Urinary biomarkers in the early detection of acute kidney injury after cardiac surgery. Clin J Am Soc Nephrol. 2009;4:873-82.

16. Han WK, Waikar SS, Johnson A, Betensky RA, Dent CL, Devarajan P, et al. Urinary biomarkers in the early diagnosis of acute kidney injury. Kidney Int. 2008;73:863-9.

17. Doi K, Leelahavanichkul A, Yuen PS, Star RA. Animal models of sepsis and sepsis-induced kidney injury. J Clin Invest. 2009;119:2868-78.

18. Shimoyama Y, Umegaki O, Agui T, Kadono N, Minami T. Neutrophil to lymphocyte ratio and platelet to lymphocyte ratio are superior to other inflammation-based prognostic scores in predicting the mortality of patients with gastrointestinal perforation. JA Clin Rep. 2017;3:49.

19. Shimoyama $Y$, Umegaki $O$, Inoue $S$, Agui T, Kadono N, Minamiet T. The neutrophil to lymphocyte ratio is superior to other inflammation-based prognostic scores in predicting the mortality of patients with pneumonia. Acta Med. 2018;72:591-3.

20. Kasymjanova G, MacDonald N, Agulnik JS, Cohen V, Pepe C, Kreisman $\mathrm{H}$, et al. The predictive value of pre-treatment inflammatory markers in advanced non-small-cell lung cancer. Curr Oncol. 2010;17:52-8.

21. Proctor MJ, Morrison DS, Talwar D, Balmer SM, Fletcher CD, O'Reilly DS, et al. A comparison of inflammation-based prognostic scores in patients with cancer. A Glasgow inflammation outcome study. Eur J Cancer. 2011;47:2633-41

\section{Publisher's Note}

Springer Nature remains neutral with regard to jurisdictional claims in published maps and institutional affiliations.
Ready to submit your research? Choose BMC and benefit from:

- fast, convenient online submission

- thorough peer review by experienced researchers in your field

- rapid publication on acceptance

- support for research data, including large and complex data types

- gold Open Access which fosters wider collaboration and increased citations

- maximum visibility for your research: over $100 \mathrm{M}$ website views per year

At $\mathrm{BMC}$, research is always in progress.

Learn more biomedcentral.com/submissions 